\title{
THE EFFECT OF SURGERY ON THE AC/A RATIO
}

\author{
E. LUCAS ${ }^{1}$, C. R. BENTLEY ${ }^{2}$ and W. A. ACLIMANDOS 3 \\ London
}

\begin{abstract}
SUMMARY
The accommodative convergence/accommodation (AC/A) ratio is used clinically as a diagnostic tool in the assessment of convergence excess and near esotropia and the differentiation of true and simulated divergence excess. An abnormally high AC/A ratio has been implicated in the aetiology of some forms of strabismus but the evidence for alteration in the ratio following surgery is inconclusive. In a prospective study of 38 patients the effect of routine squint surgery was investigated in concomitant strabismus. The $\mathrm{AC} / \mathrm{A}$ ratio was found to decrease significantly after surgery in both esotropes and exotropes. Furthermore a trend was demonstrated which suggested an increased risk of overcorrection in esotropes with a high $(\geqslant 7: 1)$ pre-operative $\mathrm{AC} / \mathrm{A}$ ratio.
\end{abstract}

The accommodative convergence/accommodation (AC/A) ratio is defined as the degree of accommodative convergence (in metre angles) exerted in response to 1 unit of accommodation.' Clinically it is possible to determine only the change in vergence associated with the stimulus to accommodate, as the centrally driven accommodative effort itself cannot be measured. ${ }^{2}$ Various methods have been employed to measure the AC/A ratio which will eliminate the influence of the other components of convergence, namely tonic, proximal and fusional convergence. The three common methods in use are the heterophoria, clinical and gradient methods. ${ }^{3-6}$

Early estimates of the normal ratio have been between $3: 1$ and $4: 1{ }^{7}$ However, later studies using the slope gradient method show the average AC/A to be 2.76 (range $0.5-$ 8.6). ${ }^{8}$ An abnormal ratio may result in excessive or insufficient accommodative convergence which, in the absence of adequate fusional reserve, will result in heterotropia or symptomatic heterophoria. Attempts to treat such cases by normalising the $\mathrm{AC} / \mathrm{A}$ ratio and therefore enabling control of the deviation have involved the use of cycloplegics. ${ }^{9}$ miotics ${ }^{10}$ orthoptic exercises ${ }^{11}$ and surgery. ${ }^{7.12-1+}$

From: 'St Thomas' Hospital, London; 'St George's Hospital, London; ${ }^{3}$ King's College Hospital, London. UK.

Correspondence to: $\mathrm{Mr}$ W. A. Aclimandos, FRCOphth. Ophthalmology Department, King`s College Hospital. Denmark Hill. London SE5 9RS, UK.
The effect of surgery on the AC/A ratio has been investigated and initially was said to improve or significantly lower an abnormal ratio, ${ }^{12}$ although other studies showed that the AC/A ratio is generally unaffected by surgery. ${ }^{7}$ More recently it has been shown that a high AC/A ratio tended to normalise whereas a low AC/A ratio may increase. ${ }^{13}$ However, these studies may be criticised since they used the clinical method to estimate the AC/A ratio. The clinical method merely subtracts the distance from the near measurement, and makes no allowance for the other components of convergence that play a role in the ocular deviation. Therefore this method cannot record a true $\mathrm{AC} / \mathrm{A}$ ratio and has also been shown to elicit higher and unpredictable results compared with the gradient method. ${ }^{3}$ The AC/A ratio determined by the gradient method and its modification by surgery has only been examined in one previous study of two esotropic patients. ${ }^{1+}$ In this study the AC/A ratio reduced from $6: 1$ and $5: 1$ to $3: 1$ and $4: 1$ respectively.

We therefore undertook a prospective study to examine the change produced by surgery on the AC/A ratio as determined by the gradient method. In particular the relationship of pre- and post-operative angle of deviation, and change in the AC/A ratio was examined in an attempt to identify any pre-operative features that might predict a successful surgical outcome.

\section{PATIENTS AND METHODS}

Patients were enrolled into the study from the population attending the Orthoptic Clinic at St Thomas' Hospital. Consecutive patients with a concomitant strabismus were included in the study. Patients with neurological disease, paralytic strabismus, a previous history of squint surgery, inability to fixate in one or both eyes due to low visual acuity and young children who could not comply with the measurements were excluded.

All patients had a full pre-operative investigation. This included visual acuity, assesment of binocular function at the appropriate distance or by using the synoptophore to elicit binocular potential, a prism cover test to a $6 / 6$ fixation target for near and distance, cycloplegic refraction and ocular examination. 
Table I. Pre-operative and post-operative results in the esotropic group

\begin{tabular}{lccc}
\hline & AC/A ratio & $\begin{array}{c}\text { Near angle } \\
\text { (prism dioptres) }\end{array}$ & $\begin{array}{c}\text { Distance angle } \\
\text { (prism dioptres) }\end{array}$ \\
\hline Pre-operative median (Q1-Q3) & $8(7-11)$ & $+35(+30,+45)$ & $+14(+8,+20)$ \\
Post-operative median (Q1-Q3) & $4(2-5)$ & $+6(-4,+12)$ & $(-4,+6)$ \\
Difference (95\% confidence interval) & $-4(-6,-3)^{*}$ & $-30(-41,-25)^{*}$ & $-10(-24,-6)^{*}$ \\
\hline
\end{tabular}

${ }^{*} p<0.0001$ (Wilcoxon rank sign test).

Table II. Pre-operative and post-operative results in the exotropic group

\begin{tabular}{lccc}
\hline & AC/A ratio & $\begin{array}{c}\text { Near angle } \\
\text { (prism dioptres) }\end{array}$ & $\begin{array}{c}\text { Distance angle } \\
\text { (prism dioptres) }\end{array}$ \\
\hline Pre-operative median (Q1-Q3) & $7(5-8)$ & $-35(-40,-20)$ & $-40(-60,-35)$ \\
Post-operative median (Q1-Q3) & $5(3-5)$ & $2(-14,+6)$ & $2(-12,+6)$ \\
Difference (95\% confidence interval) & $-2(-5,3)^{*}$ & $25(18,-60)^{* *}$ & $40(-23,-72)^{* *}$ \\
\hline
\end{tabular}

${ }^{*} p<0.0002 ;{ }^{*} p<0.001$ (Wilcoxon rank sign test).

The slope gradient method for $\mathrm{AC} / \mathrm{A}$ ratio was then performed by the same investigator on each subject preoperatively and at 2 weeks and 21 months post-operatively. The prism cover test was performed at 0.33 metre, 6 metres, and with two ophthalmic lenses (+3.00 DS and $-3.00 \mathrm{DS})$, in order that an average AC/A could be calculated. Measurement of the angle induced was taken only after the subject had fully exerted or relaxed his or her accommodation and overcome the lens strength. The amount and type of surgery and the surgeon's name were recorded.

\section{Statistical Analysis}

Data were analysed using non-parametric tests. The ratio data from the AC/A ratio and pre/post-operative angles of deviation were analysed by the Wilcoxon sign rank test. Contingency tables were constructed with appropriate groups of AC/A (low, normal, moderate, high, very high) and surgical result (success or failure), and the chi-squared test applied. In this study a surgical result was considered successful if the post-operative angles were $\leqslant 10$ dioptres of esotropia and $\leqslant 15$ dioptres of exotropia, or larger if the deviation was controlled with evidence of binocularity. The presence of a consecutive exotropia in those cases without fusion was considered to be surgical failure as this would not allow for divergence over the passage of time.

\section{RESULTS}

Twenty-five esotropes and 13 exotropes were recruited into the study. The age range was 3-10 years in the esotropic group and 7-10 years in the exotropic group, with

Table III. AC/A category comparison with surgical outcome

\begin{tabular}{lccc}
\hline AC/A ratio & Failure & Success & Total (success \%) \\
\hline Normal $(3-4)$ & 0 & 1 & $1(100 \%)$ \\
Moderate $(5-6)$ & 1 & 2 & $3(66.67 \%)$ \\
High $(\geqslant 7-10)$ & 5 & 7 & $12(58.33 \%)$ \\
Very high $(\geqslant 10)$ & 6 & 3 & $9(33.33 \%) *$ \\
Total & 12 & 13 & 25 \\
\hline
\end{tabular}

*Chi-squared $p<0.12$ (Mantel-Haenszel). an overall male to female ratio of 14:24. In the esotropic group, 20 patients were classified as partially accommodative esotropes, 1 patient was a pure convergence excess type and 4 had constant esotropias. In the exotropic group 6 were of the intermittent type with evidence of binocularity at near and the remaining 7 had constant exotropias.

Twenty patients in the esotropic group had a bi-medial recession, and 6 had unilateral recess/resect procedures. All 13 of the exotropes had unilateral medial rectus resections and lateral rectus recessions. The distribution of $\mathrm{AC} / \mathrm{A}$ ratio in the esotropic and exotropic groups pre- and post-operatively are shown in Figs. 1-4, and demonstrate a statistically significant reduction in AC/A ratio postoperatively in both the esotropic and exotropic groups (Tables I, II).

The pre-operative $\mathrm{AC} / \mathrm{A}$ ratios of the esotropic group were divided into normal, moderate, high and very high (Table III). Using chi-squared analysis a trend could be seen suggesting a greater success rate in cases with a normal AC/A ratio. To investigate this trend further we considered the surgical outcome in terms of success, failure (undercorrection), consecutive (overcorrection) and the role of fusion. In the high AC/A group there were no undercorrections and in the very high group two-thirds of patients were surgical failures. The presence of fusion appeared to improve the surgical outcome, particularly in patients with high or very high AC/A ratios (Table IV).

\section{DISCUSSION}

The results of this preliminary study show that surgical intervention produces a significant reduction in the AC/A ratio. The median decrease in ratio was 4 in esotropes and 2 in exotropes. In the esotropic group the categorisation of the $\mathrm{AC} / \mathrm{A}$ ratio in order to compare it with surgical outcome showed a trend for the high $\mathrm{AC} / \mathrm{A}$ group $(\geqslant 7: 1)$ to be overcorrected whilst in the very high $\mathrm{AC} / \mathrm{A}$ group $(\geqslant 10: 1)$ results were unpredictable. Analysis of the exotropic patients showed no statistically significant trend due to the numbers involved being too small. Ten of the 13 


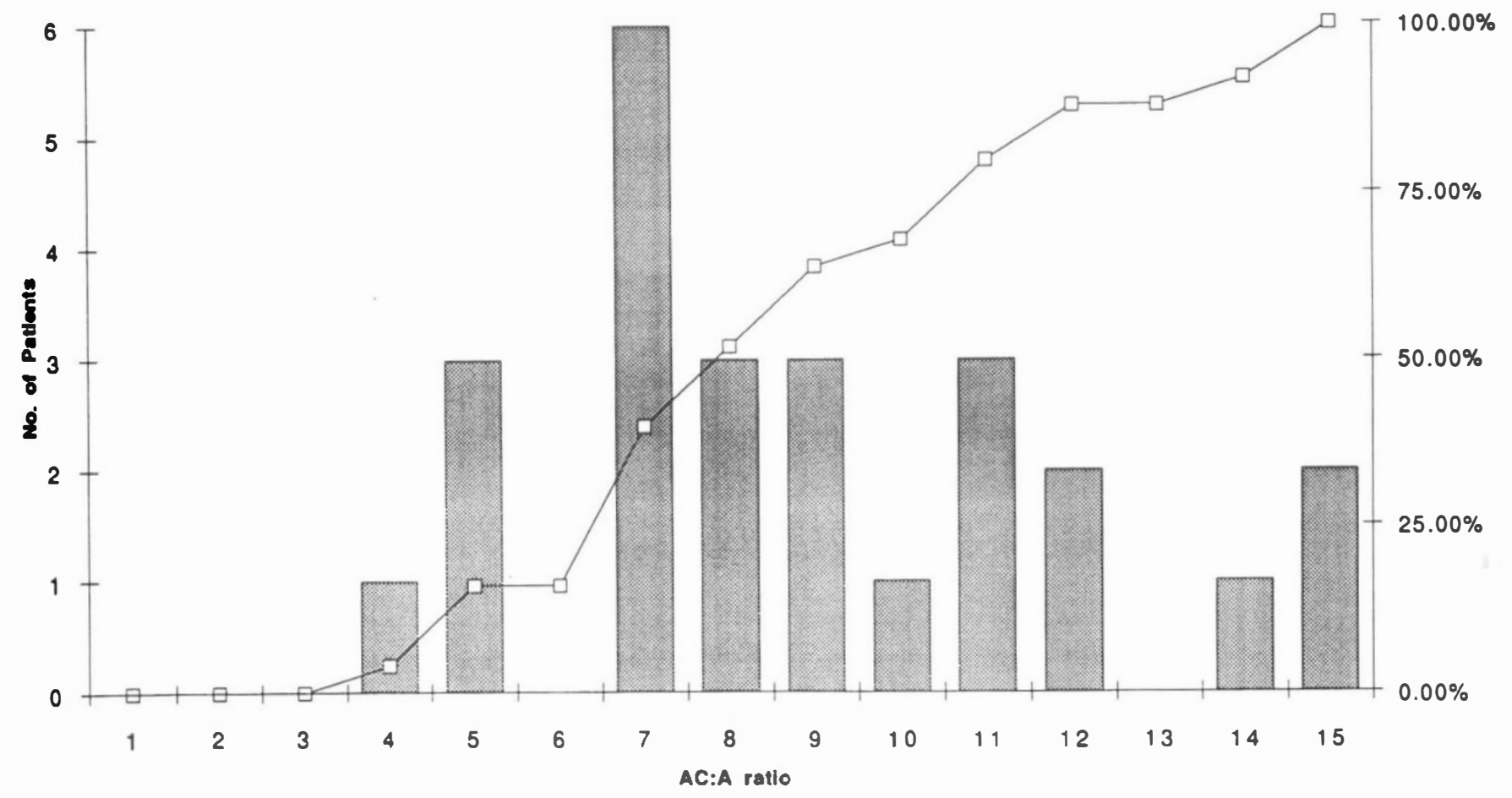

Fig. 1. Distribution of pre-operative AC/A ratio in the esotropic group. $-\square-$ Cumulative frequency.

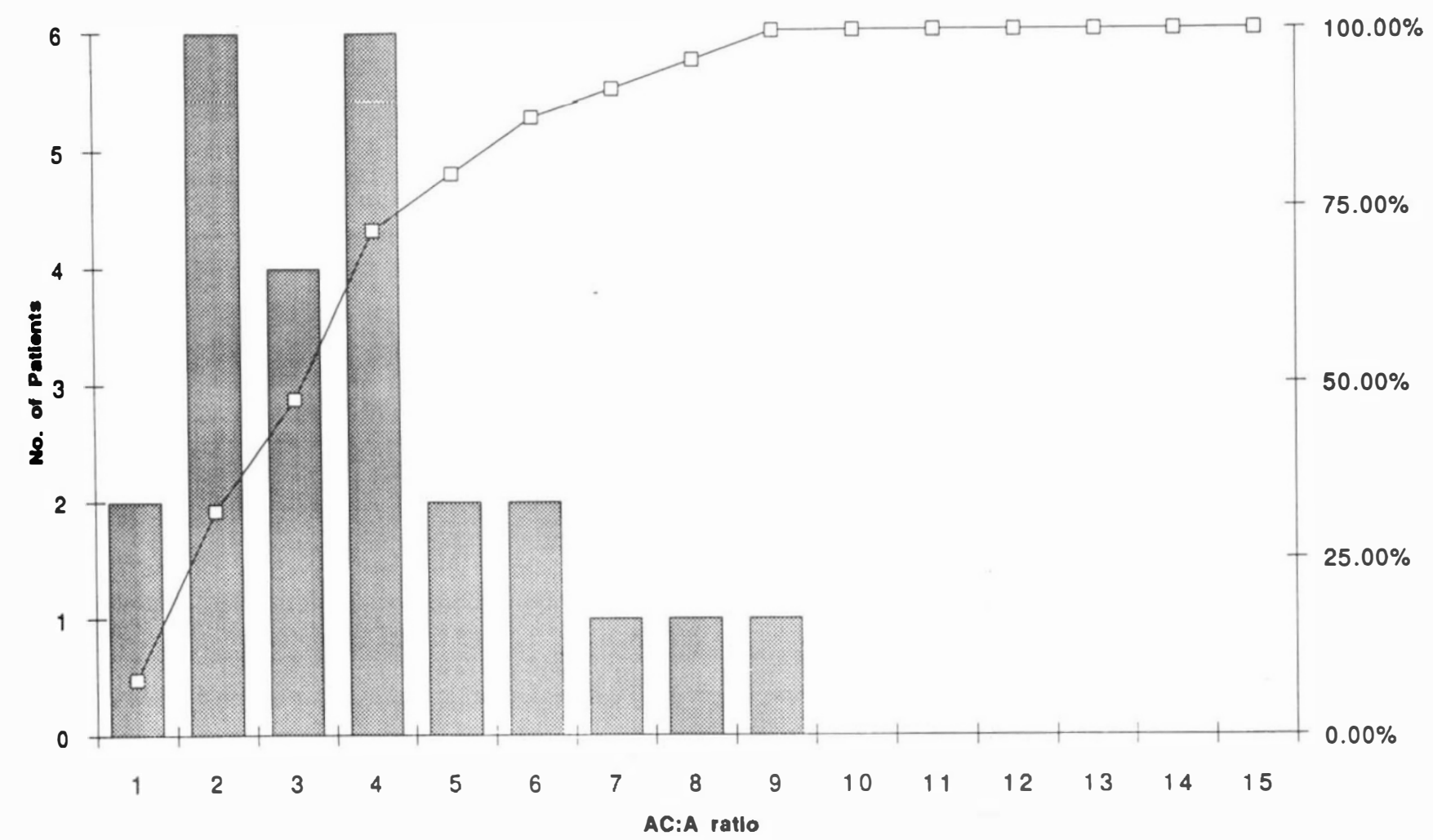

Fig. 2. Distribution of post-operative AC/A ratio in the esotropic group. $-\square-$ Cumulative frequency. 


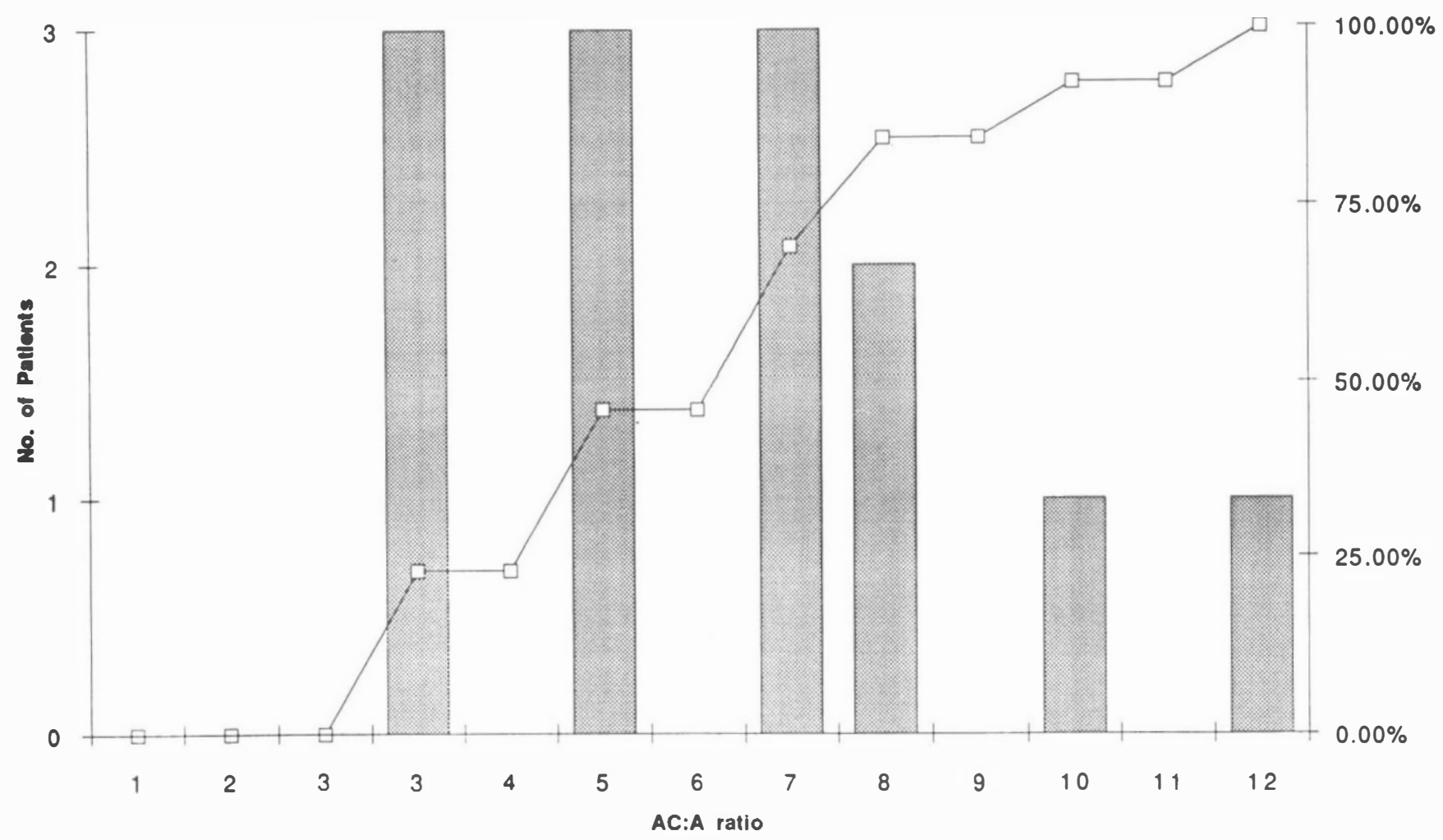

Fig. 3. Distribution of pre-operative AC/A ratio in the exotropic group. $-\square-$ Cumulative frequency.

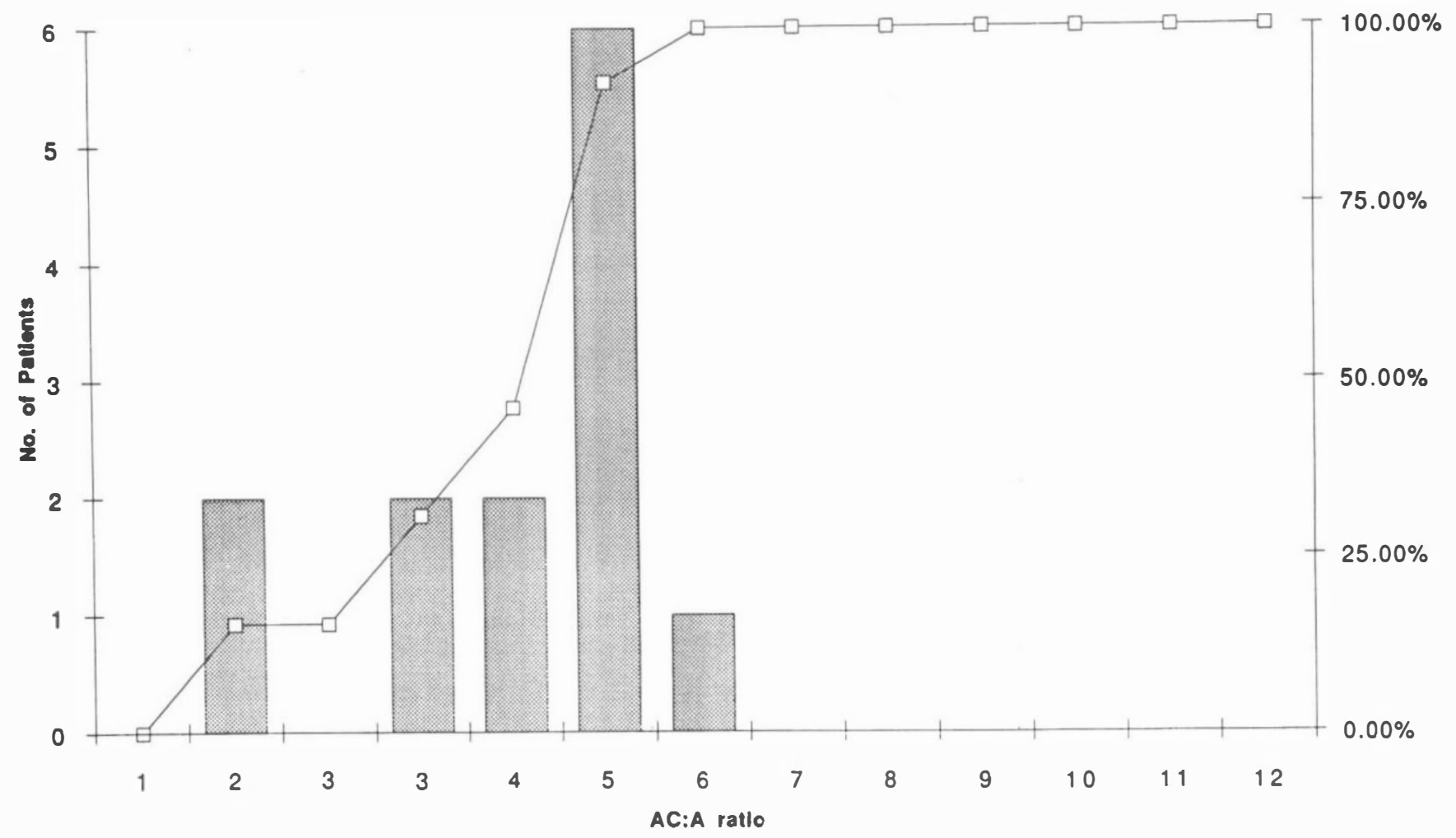

Fig. 4. Distribution of post-operative AC/A ratio in the exotropic group. $-\square-$ Cumulative frequency. 
Table IV. AC/A category comparison with consecutive outcomes and fusion

\begin{tabular}{lcccc}
\hline AC/A ratio & Success & Failure & Consecutive & Total \\
\hline Normal/moderate & $3(1)$ & $1(0)$ & 0 & $4(1)$ \\
High & $7(6)$ & 0 & $5(0)$ & $12(6)$ \\
Very high & $3(3)$ & $3(0)$ & $3(0)$ & $9(3)$ \\
Total & $13(10)$ & $4(0)$ & $8(0)$ & $25(10)$ \\
\hline
\end{tabular}

$p<0.12$ (Fisher's exact).

Numbers in brackets are patients with fusion.

cases with successful surgical outcome had evidence of fusion.

Parks ${ }^{12}$ found that surgery imposes a permanent and constant improvement upon the abnormal AC/A ratio. However, Ogle and co-workers ${ }^{7}$ felt that the $\mathrm{AC} / \mathrm{A}$ ratio was generally unaffected by surgery. Bateman and Parks ${ }^{1.3}$ found that esotropic patients with a high pre-operative $\mathrm{AC} / \mathrm{A}$ relationship were likely to undergo a normalisation of this parameter after bilateral medial rectus muscle recession. They further commented that in a subgroup of 7 patients a low pre-operative $\mathrm{AC} / \mathrm{A}$ relationship increased following surgery.

The variation in these studies investigating the change in $\mathrm{AC} / \mathrm{A}$ ratio post-operatively may simply reflect the shortcomings of the techniques used to calculate the ratio. Most of the studies ${ }^{12.13}$ used the clinical method to assess the so-called $\mathrm{AC} / \mathrm{A}$ relationship, which is not a true measure of the $\mathrm{AC} / \mathrm{A}$ ratio. The $\mathrm{AC} / \mathrm{A}$ relationship is obtained by subtracting the distance angle of deviation from the near (in prism dioptres). If the difference is 10 dioptres or more, the $\mathrm{AC} / \mathrm{A}$ relationship is said to be abnormally high. This assumes that the convergence at near is wholly exerted by the accommodation-convergence synkinesis. However, patients who have an abnormal distance-near relationship of their esotropia can be wrongly diagnosed as having a high $\mathrm{AC} / \mathrm{A}$ ratio. The ratio so determined suffers from the inaccuracies of the proximal factor as well as possible unknown elements associated with the vergence mechanism and is often higher than the $\mathrm{AC} / \mathrm{A}$ ratio as determined by the lens gradient method. Furthermore it involves an algebraic summation to the near measurement of any distance deviation due to non-accommodative factors which can lead to confusion and error. ${ }^{3}$ The gradient method used in this study gives a true estimate of the $\mathrm{AC} / \mathrm{A}$ ratio ${ }^{5.6}$ and is more accurate if more than two points are determined. The advantages of the test over the other commonly used method, namely the heterophoria method, are that it facilitates control over the accommodation exerted and can be performed for distance, thus practically eliminating proximal convergence. The change in muscle balance thus generated reflects the amount of accommodative convergence associated with a unit change of dioptric power.

The change in AC/A ratio seen in this study corroborates the work of Sears and Guber ${ }^{1+}$ who showed a reduction of the ratio in 2 patients measured by the gradient method. The reduction of the amount of accommodative convergence after medial rectus recession would suggest that weakening of the muscle has a peripheral effect upon the measurable AC/A ratio. Surprisingly this decrease also occurs with medial rectus resection in exotropes. Parks ${ }^{12}$ also comments that theoretically any surgery for exotropia should magnify an abnormal AC/A ratio since the converging muscles must be tightened. This apparent anomaly of the effect of surgical intervention on the AC/A ratio in exotropes is suggestive of an abnormal $\mathrm{AC} / \mathrm{A}$ ratio in this group being compensatory and post-operatively adjusting itself towards normal. ${ }^{12}$ It appears from the results of this study that the ratio is dependent on the ocular deviation. This is reflected by those cases in which a change from esotropia to exotropia occurred, the AC/A ratio in these cases changing from very high to low. In one exotropic patient surgery resulted in consecutive esotropia and the ratio increased from $3: 1$ to $6: 1$.

The total number of patients entered into this study was 38 , but in view of the multiplicity of clinical variables and the various types of surgery performed the number of patients in some subgroups was not sufficient to allow useful statistical analysis. Further studies are therefore required to investigate in more detail the effect of different surgical techniques and variable amounts of surgery on the $\mathrm{AC} / \mathrm{A}$ ratio.

This study has shown that the $\mathrm{AC} / \mathrm{A}$ ratio as measured by the gradient method is significantly reduced following standard bimedial recession or recess/resect procedures for both esotropic and exotropic groups. A trend has also been demonstrated which appears to suggest that there is an increasing risk of overcorrection in esotropes with a high $(\geqslant 7: 1)$ pre-operative $\mathrm{AC} / \mathrm{A}$ ratio, particularly in the absence of fusion. The importance of fusional ability at distance in preventing consecutive exotropias has been previously stressed by Rosenbaum et al. ${ }^{15}$ Patients with very high $(\geqslant 10)$ pre-operative $\mathrm{AC} / \mathrm{A}$ ratios have an unpredictable response to surgery. In patients with esotropia and a high AC/A ratio, as measured by the gradient method, conservative surgery is advisable even in the presence of relatively large near angles of deviation.

The authors thank the consultant staff of St Thomas' and King's College Hospitals for allowing examination of their patients. and the orthoptic departments of these units, in particular Ms Avery and Ms Tomlin. The authors would also like to thank Mr M. R. Stanford for help in reviewing the manuscript.

Key words: AC/A ratio. Strabismus. Surgery.

\section{REFERENCES}

1. Fry GA. An experimental analysis of the accommodationconvergence relation. Am J Optom 1937;14:402-14. 
2. Van Noorden GK. Binocular vision and ocular motility, 4th ed. St Louis: CV Mosby, 1990.

3. Breinin GM. Accommodative strabismus and the $\mathrm{AC} / \mathrm{A}$ ratio. Am J Ophthalmol 1971;71:303-11.

4. Morgan MW Jr. A comparison of clinical methods of measuring accommodative-convergence. Am J Optom 1950;27:385-96.

5. Sloan LL, Sears ML, Jablonski MD. Convergence-accommodation relationships. Arch Ophthalmol 1960;63: 283-306.

6. Martens TG, Ogle KN. Observations on accommodative convergence: especially its nonlinear relationship. Am J Ophthalmol 1959;47:455-62.

7. Ogle KN, Martens TC, Dyer JA. Ocular motor imbalance in binocular vision and fixation disparity. Philadelphia: Lea and Febiger, 1967;242.

8. Franceschetti AT, Burian HM. Gradient accommodative convergence/accommodative ratio in families with and without esotropia. Am J Ophthalmol 1970;70:558-62.

9. Christoferson KW, Ogle KN. The effect of homatropine on the accommodation-convergence association. Arch Ophthalmol 1956:55:779-91.

10. Sabin FC, Ogle KN. Accommodation-convergence association experiments with phenylephrine, pilocarpine, and physostigmine. Arch Ophthalmol 1958:59:324-32.

11. Flom MC. On the relationship of accommodation and accommodative convergence. III. Effect of orthoptics. Am J Optom 1960;37:619-32.

12. Parks MM. Abnormal accommodative convergence in squint. Arch Ophthalmol 1958;59:364-80.

13. Bateman JB, Parks MM. Clinical and computer-assisted analyses of preoperative and postoperative accommodative convergence and accommodation relationships. Ophthalmology 1981:88:1024-30.

14. Sears M, Guber D. The change in the stimulus AC/A ratio after surgery. Am J Ophthalmol 1967:64:872-76.

15. Rosenbaum AL, Jampolsky A, Scott AB. Bimedial recession in high AC/A esotropia. Arch Ophthalmol 1974;91: 251-3. 